\title{
Solvation Structure and Ion Complex Reactivity in Concentrated Aqueous Salt Solutions
}

\author{
RAMPAL, N. ${ }^{1,2}$; WANG, H.-W. ${ }^{1 *}$; BIRIUKOV, D. ${ }^{3}$; \\ Brady, A. B. ${ }^{1}$; Neuefeind, J. ${ }^{1}$; PřEdOTA, M. ${ }^{3}$; StACK, \\ A. G. ${ }^{1 *}$
}

${ }^{1}$ Chemical Sciences Division, Oak Ridge National Laboratory, Oak Ridge, TN, USA

${ }^{2}$ Department of Chemical Engineering, Columbia University, New York, NY, USA

${ }^{3}$ Institute of Physics, Faculty of Science, University of South Bohemia, Branišovská, České Budějovice, Czech Republic

The mechanisms and pathways by which mineral nucleation occurs remain hotly debated. Key to this debate is the structure and reactivity of solutes in aqueous solutions. While there are numerous existing estimates of the speciation of aqueous solutions, and their propensity to form ion pairs or larger networks of ions, the former are often indirect measures (or even fit parameters), and conflicting evidence has arisen about the latter. In this talk, I will outline our recent work to obtain more direct measures of ion pairing and complex formation using neutron diffraction with isotopic substitution on a concentrated aqueous solution. These measurements are used to calibrate effective continuum correction molecular dynamics simulations, which are then used to probe ion complex reactivity in concentrated $(4.5 \mathrm{~m})$ and dilute $(0.01 \mathrm{~m})$ solutions using a combination of rare event methods and direct simulation. The specific system is aqueous zinc chloride, chosen because it is known to contain ion pairs and other complexes of the form, $\mathrm{ZnCl}_{x}\left(\mathrm{H}_{2} \mathrm{O}\right) y^{2-x}(x$ $\leq 4$ and $y \leq 6$ ). Our results in the concentrated solution show that complex formation in this system strongly favors higher order complexes relative to existing literature estimates (e.g., $\left.\mathrm{ZnCl}_{3}{ }^{-}, \mathrm{ZnCl}_{4}{ }^{2-}\right)$ but that the relative number of coordinated waters is variable. More importantly, within the concentrated solution we found that the local environment is dominated by solvent-separated ion complexes. Relative to the dilute solution, these are found to increase the free energy of activation for formation of individual ion complexes, sometimes change the mechanism of complex formation, and enhance the thermodynamic stability of complexes. We demonstrate that the equilibrium constants for a given ion complex are sensitive to the extended solvation environment, which makes the "constant" in fact dependent on concentration. Other reactions, such as the nucleation of solid phases, are likely to be affected in a similar manner. 